\title{
An asymptotic Strategy to Couple Homogenized Elastic Structures
}

\author{
Alexander G. Kolpakova*, Igor V. Andrianov ${ }^{\mathrm{b}}$, Sergei I. Rakin ${ }^{\mathrm{c}}$ and \\ Graham A. Rogerson ${ }^{\mathrm{d}}$
}

\footnotetext{
$a^{*}$ Siberian State University of Telecommunication and Informatics, Kirov str. 86, RU630009, Novosibirsk, Russia; corresponding author,e-mail: algk@ngs.ru

${ }^{b}$ Institute of General Mechanics, RWTH Aachen University, Templergraben 64, D-52056, Aachen, Germany

${ }^{c}$ Siberian Transport University, Dusi Kovalchuk str. 191, RU-630049, Novosibirsk, Russia

${ }^{d}$ School of Computing and Mathematics, Keele University, Keele, Staffordshire. ST5 5BG. $U K$
}

Abstract. A two-scale methodology to calculate the local stress-strain state (SSS) in structures composed of connected elements is proposed. The methodology is based on the assumption that the connecting unit has a size small in comparison to the objects being connected. It is demonstrated that the problem of connection allows asymptotic decomposition. At the macroscopic level (the zero order approximation), an interface problem, with appropriate interface conditions, is revealed. At this order, the individual properties of the joint are neglected. These properties manifest themselves at the next asymptotic order, which takes into account all individual joint properties using the solution of the macroscopic problem. The local SSS in the vicinity of joint consists of the SSS in the connecting unit, together with rapidly decaying boundary layers in the connected elements. A detail elucidation of the local SSS in the connecting unit is an important distinction of this work from previous studies of connected structures. Motivated by the asymptotic analysis, a numerical method for simultaneously calculating the SSS in both the connected structures and the connecting unit is developed. An illustrative example, involving computation of the SSS in the vicinity of an explosion welding seam, is presented.

Keywords: elasticity theory; joint; macroscopic level; microscopic level; asymptotic decomposition.

\section{Introduction. Models of joint - interface surface or transition zone}

Structural components composed of connected elements are common place in engineering practice. Two basic approaches, both based on models involving an interface surface and 
connecting unit or transition zone, are typically employed to determine mechanical response. In the first, the two elements of the structure are assumed to be directly adjacent to some interface surface, described by an "interface condition" on the surface. Often ideal contact conditions are used, which in the elastic case take the form

$$
[\mathbf{u}]=0,\left[\boldsymbol{\sigma}_{n}\right]=0,
$$

where [] denotes a jump on the interface surface, $\mathbf{u}$ is the displacement vector and $\boldsymbol{\sigma}_{n}$ is the stress vector normal to the interface surface. These conditions are often formulated on a weld seam and have also been used for riveted and glued joints [8, 9, 10]. This approach can be oversimplified: for example, as conditions (1) do not contain parameters of the actual weld, a weld seam with a complex physical and geometric structure can only be treated as a surface without any individual properties. However, this idealization, by virtue of its simplicity, has been widely used and still proved to be useful for calculating the stress-strain state (SSS) in connected structural elements and within the vicinity of the joint. A second approach involves a detailed study of the interconnecting unit (e.g. the bolts in a bolted connection) or transition zone (e.g. welded seam) within the framework of the spatial elasticity theory problem [21, 25, 26]. This approach allows one to take into account the physical and geometric characteristics of the connection and calculate the SSS in all structural elements. In this case, the connecting unit is often regarded as an isolated and independent element, not associated with the entire structure.

When trying to treat a connecting unit as part of the entire structure, we arrive at the following problem. Denote by $u_{0}^{-}(\mathbf{x})$ and $u_{0}^{+}(\mathbf{x})$, displacements in the main parts of the connected structural elements, i.e. in the parts of connected structural elements placed far from the joint. These solutions usually have simple form.

The solutions $u_{0}^{-}(\mathbf{x})$ and $u_{0}^{+}(\mathbf{x})$ usually do not satisfy conditions (2). This discrepancy is compensated for by the introduction of boundary layers (BLs) $\zeta^{-}(\mathbf{x})$ and $\zeta^{+}(\mathbf{x})$, which exhibit rapid decay away from the interface surface in domains $P^{-}$and $P^{+}$, respectively. This corresponds to an idealized contact scheme suitable for some practical situations, for example, when two elements are connected by compression (diffusion welding). The mathematical technique for coordinating the functions $u_{0}^{-}(\mathbf{x})+\zeta^{-}(\mathbf{x})$ and $u_{0}^{+}(\mathbf{x})+\zeta^{+}(\mathbf{x})$, and for the satisfaction of the contact condition, is well developed (see, e.g. [4], and references herein). In respect of the interface problem for composites, several authors have addressed this problem, see for example [1, 6, 12, 21, 23, 28] among others.

In the connecting unit, or transition zone model, there is a region $P_{0}$ between the elements $P^{-}$and $P^{+}$, within which the solution of the problem has the form: 


$$
\begin{gathered}
u(\mathbf{x})=u_{0}^{-}(\mathbf{x})+\zeta^{-}(\mathbf{x}) \text { at } P^{-} ; \\
u(\mathbf{x})=\varphi(\mathbf{x}) \text { at } P_{0} ; \\
u(\mathbf{x})=u_{0}^{+}(\mathbf{x})+\zeta^{+}(\mathbf{x}) \text { at } P^{+} .
\end{gathered}
$$

The elasticity theory problem for the functions (2) can be solved separately in each domain $P_{0}, P^{-}$and $P^{+}$, with subsequent matching of the solutions. However, a simpler way is to employ the boundary perturbation method and the method of local perturbations [23,24,25]. We briefly describe the main idea of this approach.

In many cases, the sizes of connecting units, or transition zones, are small in comparison to the size of the connected structural elements. Let us denote the relative size of the connecting units by $\varepsilon$. The local perturbation theory is based on representation of the solution of the elasticity problem in the form

$$
\mathbf{u}^{0}(\mathbf{x})+\varepsilon \mathbf{v}(\mathbf{x}, \mathbf{x} / \varepsilon)
$$

In (3), $\mathbf{u}^{0}(\mathbf{x})$ is the smooth leading order solution and $\varepsilon \mathbf{v}(\mathbf{x}, \mathbf{x} / \varepsilon)$ its corrector. In our approach, the corrector $\mathbf{v}(\mathbf{x}, \mathbf{y})$ takes the form of a local perturbation. We call a function "the local perturbation, corresponding to a perturbation of the geometry of the domain or material property of material occupying the domain", if it tends to zero as the distance between the actual point and the aforementioned domain tends to infinity.

Note that Ansatz (3) is well known from homogenization theory [3, 15, 27]. However, in homogenization theory, the corrector is periodic in $\mathbf{y}$. This difference leads to drastic differences in the limit models.

The local perturbation method, on the one hand, explains the difference between the interface model and the connective unit model (transition zone), and on the other hand, demonstrates the consistency of the models. The interface model describes the global behavior of the solution, the model of connecting unit (transition zone) also describes the local perturbation of the solution. The interface model solution, obtained using the relative size of the transition zone as a small parameter, is the first term of the asymptotic solution of the original problem. This explains the effectiveness of the interface model for the analysis of the macroscopic behavior of connected bodies despite the neglecting a detailed analysis of the interface. A similar, but not identical problem arises in the multi-element thin-walled structures (see, e.g. [18, 24, 31, 32]), where the structural mechanics model occurs within the zero-term of the asymptotic solution to the elasticity theory problem. 
The paper is organized as follows. In Sect. 2, we develop an asymptotic analysis for homogeneous joined bodies. Sect. 3 illustrates an application of the local perturbation method to the calculation of the local SSS in a welded seam arising as a result of explosion welding of homogeneous materials. In Sect. 4, we expand the local perturbation method to the case of inhomogeneous (composite) joined bodies. Sect. 5 illustrates the application of the local perturbation method to the calculation of local SSS in a welded seam arising as a result of explosion welding of homogeneous and composite materials. In Sect. 6, we determine the minimal fragment of the connected elements adjacent to the connecting unit needed so that the solution in the selected zone approximates the solution of the original problem with sufficient accuracy. Lattice-solid connection is studied in Sect. 7. Finally, some concluding remarks are presented in Sect.8.

\section{Asymptotic analysis for the homogeneous joined bodies}

Let us consider a 2D or 3D elasticity problem for connected inhomogeneous elements occupying the domain $P=P^{-} \cup P_{0} \cup P^{+}$. It is assumed that the elements, or connecting zones, are of a periodic structure. We denote by $\varepsilon$ the relative size of inhomogeneties (the characteristic dimension of the inhomogeneties divided by the characteristic size of the connected elements), see Fig.1. For the types of scenario envisaged, it is assumed that $\varepsilon<<1$.

The variational formulation of the problem is

$$
\int_{P} a_{i j k l}(\mathbf{x} / \varepsilon) u_{i, j}(\mathbf{x}) v_{k, l}(\mathbf{x}) d \mathbf{x}=\int_{P} \mathbf{q}(\mathbf{x}) \mathbf{v}(\mathbf{x}) d \mathbf{x}, \text { for any } \mathbf{v} \in V
$$

see [7,30], where $a_{i j k l}(\mathbf{x} / \varepsilon)$ are local elastic constants, $\mathbf{u}(\mathbf{x})$ is the displacement vector, $\mathbf{v}(\mathbf{x})$ is a trial function, $V$ is the set of admissible displacements (namely, $V$ is the set of functions belonging to $H_{2}^{1}(P)$ with the proper boundary conditions) and $\mathbf{q}(\mathbf{x})$ is the mass force vector. Generally, the local elastic constants are highly oscillating functions; however, if the connected body is homogeneous, $a_{i j k l}$ are constant.

We consider the "non-degenerated" joints, i.e. joints for which the elastic characteristics are similar to the characteristics of the joined structures.

The connecting zone between two structures necessitates both microscopic and macroscopic consideration. If the interface of the bodies is uniform (for example, rectilinear), not only from a macroscopic, but also from a microscopic point of view, we have a standard (one-scale) interface problem within the theory of inhomogeneous elasticity. However, within the rectilinear "in the 
rough" approximation (viewed by the "naked eye"), the boundary is usually not straight on a fine scale (viewed by the "microscope").
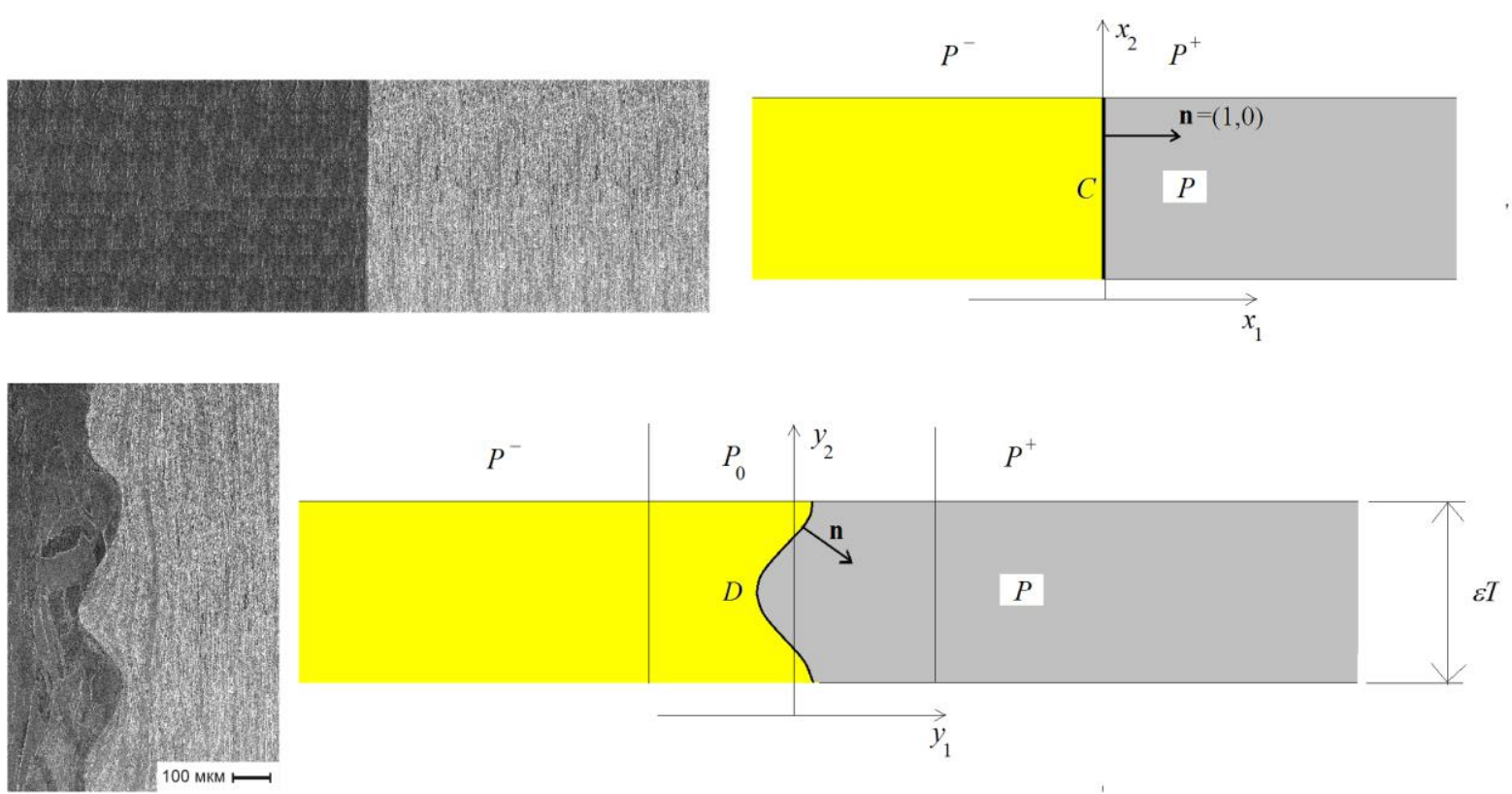

Fig.1. Macroscopic (top) and microscopic (bottom) view of seam caused by explosion welding.

The global elastic constants of the connected homogeneous bodies displayed in Fig.1 (top) are described by the step function

$$
a_{i j k l}^{0}(\mathbf{x})=\left\{\begin{array}{l}
a_{i j k l}^{-} \text {for } x_{1}<0 \\
a_{i j k l}^{+} \text {for } x_{1}>0 .
\end{array}\right.
$$

The local elastic constants of the connected homogeneous bodies displayed in Fig.1 (bottom) are described by step function

$$
a_{i j k l}(\mathbf{y})=\left\{\begin{array}{l}
a_{i j k l}^{-} \text {on the left from the interface surface } D, \\
a_{i j k l}^{+} \text {on the right from the interface surface } D .
\end{array}\right.
$$

We remark that the global $a_{i j k l}^{0}$ and local $a_{i j k l}$ elastic constants are different in the thin vicinity of the joint.

Macroscopic deformations are determined from the solution to the elastic interface problem for the structure "as a whole", for which the elastic constants (5) are appropriate. These deformations can be considered locally constant, but only in bodies to the left and right of the interface. When passing through the boundary, the overall strains $e_{\alpha \beta}(\mathbf{x})$ undergo a jump and may be described by the step function 


$$
e_{\alpha \beta}(\mathbf{x})=\left\{\begin{array}{l}
e_{\alpha \beta}^{-} \text {for } x_{1}<0 \\
e_{\alpha \beta}^{+} \text {for } x_{1}>0
\end{array}\right.
$$

Following [16, 17], we seek the solution of the BVP (4) in the form (3).

We now introduce the "fast" variable $\mathbf{y}=\left(y_{1}, y_{2}\right)=\mathbf{x} / \varepsilon$ and assume that the corrector $\mathbf{u}_{1}(\mathbf{y})$ is " a local perturbation", i.e.

$$
\mathbf{u}_{1}(\mathbf{y}) \rightarrow 0 \text { as }\left|y_{1}\right| \rightarrow \infty \text { and periodic with period } T \text { along } y_{2} .
$$

Let us explain condition (8). Stresses corresponding to deformations (7) satisfy equilibrium equations both in macro (Fig.1, top) and in the micro fragment of the material (Fig.1, bottom) to the left and to the right of the interface. For a macro problem, one can always choose $e_{\alpha \beta}^{-}$ and $e_{\alpha \beta}^{+}$in such a way that the equilibrium conditions on the macroscopic interface $S$, are satisfied: $a_{i 1 k l}^{-} e_{k l}^{-}=a_{i 1 k l}^{+} e_{k l}^{+}:$(normal $\mathbf{n}=(1,0)$, see Fig.1, top).

We remark that if we consider the same stresses on a microscopic interface $D$, in general, $a_{i j k l}^{-} e_{k l}^{-} n_{j} \neq a_{i j k l}^{+} e_{k l}^{+} n_{j}$, because of the variability of the normal vector $\mathbf{n}$ on $D$, see Fig. 1 . In this case, the mean value of the discrepancy $a_{i j k l}^{-} e_{k l}^{-} n_{j}-a_{i j k l}^{+} e_{k l}^{+} n_{j}$ is non-zero, the average values of $a_{i j k l}^{-} e_{k l}^{-} n_{j}$ and $a_{i j k l}^{+} e_{k l}^{+} n_{j}$ along $D$ are equal to $a_{i 1 k l}^{-} e_{k l}^{-}$and $a_{i 1 k l}^{+} e_{k l}^{+}$. This follows from zeroing out of the integral of the normal vector $\mathbf{n}$ along the closed contour and compensating for the discrepancy by applying fictitious stresses on $D$; these stresses have a zero mean value along $D$. In accordance with the Saint-Venant principle (see its justification for a strip in [22]), this compensation will give rise to a boundary layer that rapidly decays away from $D$, in our case as $\left|y_{1}\right| \rightarrow \infty$

We denote by $V_{1}=\left\{\mathbf{u}_{1}(\mathbf{y}) \in H_{2}^{1}(P): \mathbf{u}_{1}(\mathbf{y})\right.$ satisfies (8) $\}$ the set of possible perturbations. This set of functions is useful for our aim. On the one hand, $V_{1}$ contains decay functions which describe the BLs outside the joint; on the other hand, $V_{1}$ contains the functional class $H_{2}^{1}\left(P_{0}\right)$, allowing us to describe arbitrary deformations in the domain $P_{0}$ occupied by the connecting unit and, thus, describe the SSS in the connecting units of an arbitrary complex structure.

Ansatz (3) has some similarities to the one used in homogenization theory $[3,5,13]$. There are however two principal differences between the correctors used in homogenization theory and those considered here:

1. In homogenization theory, the corrector is assumed to be a periodic function, while the local perturbation $\mathbf{u}_{1}(\mathbf{y})$ vanishes with distance from the joint. 
2. In homogenization theory, the homogenized solution $\mathbf{u}_{0}(\mathbf{x})$ to the limit problem has continuous derivatives; whereas in the case under consideration the derivatives of $\mathbf{u}_{0}(\mathbf{x})$ have jumps. The periodicity of the corrector and the continuity of derivatives of $\mathbf{u}_{0}(\mathbf{x})$ are essential in homogenization theory $[3,5,13]$ and influence both the analysis and final result. The trial function has similarity to the $\mathbf{u}(\mathbf{x})$ in Ansatz (3) and will be assumed in the form

$$
\mathbf{v}(\mathbf{x})=\mathbf{v}_{0}(\mathbf{x})+\varepsilon \mathbf{v}_{1}(\mathbf{x} / \varepsilon)
$$

where $\mathbf{v}_{1}(\mathbf{y}) \in V_{1}$ (i.e. $\mathbf{v}_{1}(\mathbf{y}) \rightarrow 0$ as $\left|y_{1}\right| \rightarrow \infty$ and is periodic with period $T$ along $y_{2}$ ).

Using Ansatzes (3), (9), together with equation (4), one obtains the problem for "the local corrector" $\mathbf{u}_{1}(\mathbf{y})$

$$
\int_{P} a_{i j k l}(\mathbf{x} / \varepsilon)\left(u_{1 k, l y}+u_{0 k, l x}\right) v_{1 i, j y} d \mathbf{x}=0 \text { for any } \mathbf{v} \in V_{1} .
$$

Summation over repeating indices is assumed unless otherwise indicated.

In obtaining (10), we have used the well-known differentiation rule [27]

$$
\frac{\partial}{\partial x_{i}} f(\mathbf{x}, \mathbf{x} / \varepsilon)=f,_{i x}(\mathbf{x}, \mathbf{y})+\varepsilon^{-1} f,_{i y}(\mathbf{x}, \mathbf{y}) .
$$

The variational equation (10) is equivalent to the BVP

$$
\left\{\begin{array}{l}
\left(a_{i j k l}(\mathbf{y}) u_{1 k, l y}+a_{i j \alpha \beta}(\mathbf{y}) u_{0 \alpha, \beta x}(\mathbf{x})\right)_{, j y}=0 \text { in } P, \\
\left(a_{i j k l}(\mathbf{y}) u_{1 k, l y}+a_{i j \alpha \beta}(\mathbf{y}) u_{0 \alpha, \beta x}(\mathbf{x})\right) n_{j}=0 \text { on } S, \\
\mathbf{u}_{1}(\mathbf{y}) \text { is periodic in } y_{2} \text { with period } T ; \mathbf{u}_{1}(\mathbf{y}) \rightarrow 0 \text { as }\left|y_{1}\right| \rightarrow \infty,
\end{array}\right.
$$

where $S$ denotes a "free" surface of the periodicity cell $P$ (if $P$ contains holes) and $\mathbf{n}$ is the unit outer normal vector.

In the standard homogenization procedure solution of the homogenized problem represented by the continuous functions of the slow variable. In BVPs describing cell problems they can be treated as constants [27]. In our case, the functions $u_{0 \alpha, \beta x}(\mathbf{x})$ may have a jump, making impossible to separate the fast and slow variables in the manner typically used in the homogenization theory.

The possible discontinuities of the functions $u_{0 \alpha, \beta x}(\mathbf{x})$ leads to another significant difference with homogenization theory, namely, the BVP (11), (12) is not solvable for arbitrary $u_{0 \alpha, \beta x}(\mathbf{x})$. Let us derive the solvability condition for the BVP (11), (12). Integrating equation (11) by parts, and taking into account the boundary conditions (12), we obtain

$$
\int_{y_{1}=-\infty} \boldsymbol{\sigma}_{n} d y_{2}+\int_{y_{1}=+\infty} \boldsymbol{\sigma}_{n} d y_{2}=0
$$


In (13) $\boldsymbol{\sigma}_{n}=\sigma_{i 1} \mathbf{e}_{i}$ denotes the vector of normal stresses on the cross-section $y_{1}=$ const. The normal stresses $\boldsymbol{\sigma}_{n} \rightarrow a_{i 1 k l}^{-} e_{k l}^{-} \mathbf{e}_{i}$ as $y_{1} \rightarrow-\infty$ and $\boldsymbol{\sigma}_{n} \rightarrow a_{i 1 k l}^{+} e_{k l}^{+} \mathbf{e}_{i}$ as $y_{1} \rightarrow \infty$, where $e_{\alpha \beta}^{-}\left(e_{\alpha \beta}^{+}\right)$are the strains corresponding to the displacements $\mathbf{u}_{0}(\mathbf{x})$ in the "main part" (left and right) of the structure on the left (right) of the joint. From equation (13), it follows that

$$
a_{i 1 \alpha \beta}^{-} e_{\alpha \beta}^{-}=a_{i 1 \alpha \beta}^{+} e_{\alpha \beta}^{+}(i=1,2,3) .
$$

Equality (14) is the solvability condition for the BVP (11), (12), which confirms the impossibility of separating the fast and slow variables within the BVP (11), (12). However, if we use condition (14) to express $e_{\alpha \beta}^{-}$through $e_{\alpha \beta}^{+}$(or vice versa), we will arrive at a problem in which the separation of fast and slow variables is possible, but in a different manner to that typically encountered. For our purposes, the BVP (11), (12) is convenient and we will carry out the aforementioned transformations of this problem here.

The appearance of compatibility conditions when a wavy boundary is homogenized has been previously noted and discusses in respect of a free boundary, see [27]. The problem analyzed in [27] and the problem currently under consideration are different. It is clearly seen that the compatibility conditions arising in [27] directly take into account the geometric properties of the boundary, whilst in our case, only the solutions of the limit problems enter into the compatibility conditions, with the individual properties of the interface not entering at all.

For orthotropic materials the equality (14) takes the form

$$
a_{i 1 \alpha 1}^{-} e_{\alpha 1}^{-}=a_{i 1 \alpha 1}^{+} e_{\alpha 1}^{+} \text {. }
$$

In consequence, for orthotropic materials the solvability condition for BVP (11), (12) contains overall deformations with indices of the form $\alpha 1$ (i.e., 11 and 21). On the other hand, when we consider the BVP (11), (12), with deformation along the $O y_{2}$ axis allowed (indices $\alpha \beta=22$ ), the solvability condition does not arise.

We now describe the phenomenon of "erasing" of the individual properties of the joint. Substituting into (4) functions $\mathbf{u}(\mathbf{x})=\mathbf{u}_{0}(\mathbf{x})+\varepsilon \mathbf{u}_{1}(\mathbf{x} / \varepsilon)$ and $\mathbf{v}(\mathbf{x})=\mathbf{v}_{0}(\mathbf{x})+\varepsilon \mathbf{v}_{1}(\mathbf{x} / \varepsilon) \quad$ one obtains

$$
\int_{P} a_{i j k l}(\mathbf{x} / \varepsilon)\left(u_{1 k, l y}+u_{0 k, l x}\right)\left(v_{1 k, l y}+v_{0 k, l x}\right) d \mathbf{x}=\int_{P} \mathbf{q}(\mathbf{x}) \mathbf{v}(\mathbf{x}) d \mathbf{x} \text { for any } \mathbf{v} \in V .
$$

Consider the terms 


$$
\begin{gathered}
\int_{P} a_{i j k l}(\mathbf{x} / \varepsilon) u_{0 k, l x}(\mathbf{x}) v_{1 k, l y}(\mathbf{x} / \varepsilon) d \mathbf{x}+\int_{P} a_{i j k l}(\mathbf{x} / \varepsilon) u_{1 k, l y}(\mathbf{x} / \varepsilon) v_{0 k, l x}(\mathbf{x}) d \mathbf{x} \\
+\int_{P} a_{i j k l}(\mathbf{x} / \varepsilon) u_{1 k, l y}(\mathbf{x} / \varepsilon) v_{1 k, l y}(\mathbf{x} / \varepsilon) d \mathbf{x}
\end{gathered}
$$

arising after expanding parenthesis in (16). Expressed in the variables $\mathbf{y}=\mathbf{x} / \varepsilon$, expression (17) takes form

$$
\begin{gathered}
\varepsilon^{n} \int_{\varepsilon^{-1} P} a_{i j k l}(\mathbf{y}) u_{0 k, l x}(\varepsilon \mathbf{x}) v_{1 k, l y}(\mathbf{y}) d \mathbf{y}+\varepsilon^{n} \int_{\varepsilon^{-1} P} a_{i j k l}(\mathbf{y}) u_{1 k, l y}(\mathbf{y}) v_{0 k, l x}(\varepsilon \mathbf{x}) d \mathbf{y} \\
+\varepsilon^{n} \int_{\varepsilon^{-1} P} a_{i j k l}(\mathbf{y}) u_{1 k, l y}(\mathbf{y}) v_{1 k, l y}(\mathbf{y}) d \mathbf{y}
\end{gathered}
$$

where $\varepsilon^{-1} P=\left\{\mathbf{y}=\varepsilon^{-1} \mathbf{x} \in P: \mathbf{x} \in P\right\}$. In terms of $\mathbf{y}=\varepsilon^{-1} \mathbf{x}$, the domain $\varepsilon^{-1} P$ is large and its volume estimated as $\varepsilon^{-n}$. The functions $\mathbf{u}_{1}(\mathbf{y})$ and $\mathbf{v}_{1}(\mathbf{y})$ are localized near the connections in the domain, for which the volume is estimated as $\varepsilon^{-n+1}(2 H+D)$. Here $H$ denotes the "depth of penetration" of the BLs to the left and right subdomains, $\varepsilon^{-1} P^{-}$and $\varepsilon^{-1} P^{+}$in the direction $x_{1}$, and $D$ the size of $P_{0}$ in the same direction. Since $H$ and $D$ are finite, the sum (18) tends to zero as $\varepsilon \rightarrow 0$.

For the similar reason to those noted above, we can change the coefficients $a_{i j k l}(\mathbf{y})$ in (6) for those in (5), after this change equation (16) takes the form

$$
\int_{P} a_{i j k l}^{0}(\mathbf{x}) u_{0 k, l x}(\mathbf{x}) v_{0 k, l x}(\mathbf{x}) d \mathbf{x}=\int_{P} \mathbf{q}(\mathbf{x}) \mathbf{v}(\mathbf{x}) d \mathbf{x} \text { for any } \mathbf{v} \in V .
$$

Note that for a body of periodic structures (i.e. in homogenization theory) the integrands in (18) are not localized but periodic and, as a result, the sum (18) is finite as $\varepsilon \rightarrow 0$, leading to the arising of homogenized constants.

The variational equation (19) determines the first term of the asymptotic expansion (3) and is equivalent to a problem with the interface conditions (1). In this case, the properties of the joint do not influence on solution.

The local stress is $\sigma_{i j}=a_{i j k l}(\mathbf{x} / \varepsilon) u_{k, l}(\mathbf{x})$ and, using expression (3), we obtain

$$
\sigma_{i j}=a_{i j k l}(\mathbf{y}) u_{1 k, l y}+a_{i j \alpha \beta}(\mathbf{y}) u_{0 \alpha, \beta x}(\mathbf{x}),
$$

where $\mathbf{u}_{1}(\mathbf{y})$ is the solution to the problem (11), (12), this problem directly depending on the properties of the joint. Thus, we can conclude that the interface model is the first approximation of the connecting unit (transition zone) model. 


\section{Example 1. Stress concentration in the seam formed by explosion welding of homogeneous materials}

We now apply the developed method to a computation in respect of an explosion welding seam. In the process of explosion welding the transition zone between the connecting materials is very thin and to a first approximation may be neglected. Microscopically an explosion welding results in a transformation of the right linear boundaries of the welding bodies into a wavy interface surface. The resulting wavy interface surface has geometry close to periodic. This periodic structure plays the role of an interface unit.

We consider a seam arising from the explosion welding of copper (on the left) and aluminum (on the right), see Fig.1. The mechanical characteristics of the materials are:

$$
\begin{gathered}
\text { copper }-E=100 \mathrm{Gap}, v=0.35, \\
\text { aluminum }-E=70 \mathrm{Gap}, v=0.34 .
\end{gathered}
$$

We carry out computations for the wavy seam displayed in Fig.1, with the shape of the seem approximated as

$$
x_{1}=\varepsilon A \sin \left(x_{2} / \varepsilon\right)
$$

with

$$
\varepsilon \approx 70 \cdot 10^{-4} \mathrm{~m} \text { (70 micron), } A=0.8 \text {. }
$$

The calculations are carried out in respect of plane strain problem.

The problem was solved numerically using the ANSYS FEM code. Theoretical analysis shows the necessity for a numerical solution to satisfy the solvability condition (14). The computational procedure used here and in Sect. 5 is based on the notion of a representative element for the joint which is discussed in detail in Sect.6. Solutions were obtained for all basis types of plane deformations - uniform stretching along $O x$-axis ( $O y$-axis) and a shear-type deformation.

Figs 2-4 present the von Mises stress $\sigma=\sqrt{\sigma_{1}^{2}-\sigma_{1} \sigma_{2}+\sigma_{2}^{2}}\left(\sigma_{1}, \sigma_{2}\right.$ are the principal plane stresses) for basic types of plane strain $e_{\alpha \beta}=\delta_{\alpha \beta}, \alpha, \beta=1,2$ We select for presentation the von Mises stress because for metals the von Mises strength criterion $\sigma \leq \sigma^{*}$ is often used [8]. 


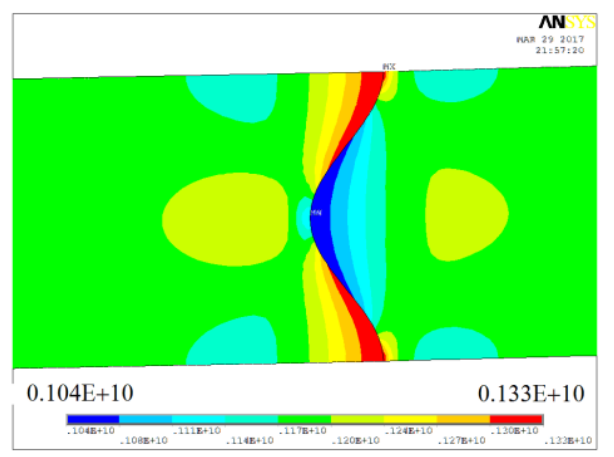

Fig.2. Local stress corresponding to the overall shear (deformed periodicity cell and local von Mises stress) (values on the scale vary from $0.1046 E+10$ on ths to $0.133 E+10$ on rhs with step 0.003-0.004E+10)

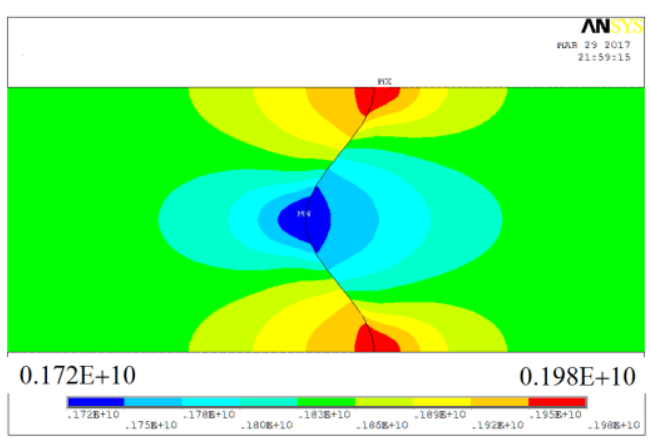

Fig.3. Local stress corresponding to the overall extension along Ox-axis (deformed periodicity cell and local von Mises stress) (values on the scale vary from on ths $0.172 E+10$ to $0.198 E+10$ on rhs with step $3 E+10$, the first step is $0.03 E+10)$

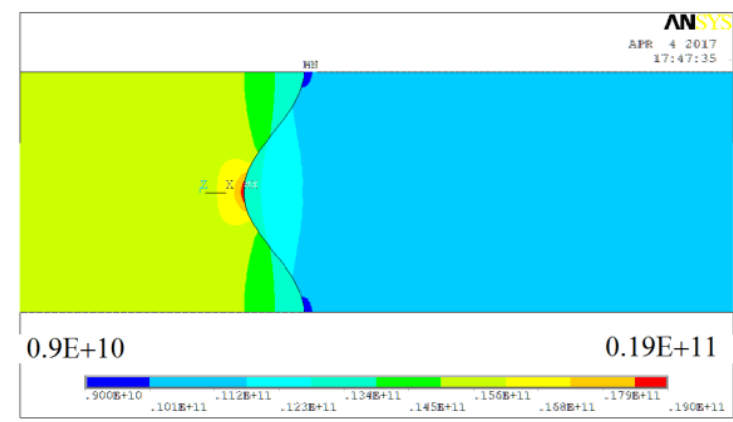

Fig.4. Local stress corresponding to the overall extension along Oy-axis (deformed periodicity cell and local von Mises stress) (values on the scale vary from $0.9 E+10$ on lhs to $0.19 E+11$ on rhs with step $0.011 E+11$ ).

We remark that solutions corresponding to the overall shear and overall extension along $O x$ axis may be obtained only if the overall strains satisfy the solvability condition. In Fig.2-4, both the SSS in the vicinity of the wavy boundary and decaying BLs are depicted. The vicinity of the wavy boundary may be treated as a joint unit. The SSSs in the BLs and that in the vicinity of the 
wavy boundary have a different appearance; it decays in the BLs and follows to the shape of the boundary in the vicinity of the wavy boundary. The maximum von Mises stress is localized at the top of the half-wave. We also note that the BLs decay at a distance 3-5 $\varepsilon T$ from the boundary. This estimate of the BLs decay zone coincides with that obtained in [25] for BLs in thermal problems.

\section{Asymptotic analysis for the inhomogeneous joined bodies}

In many modern designs, connected structural elements consist of composite materials [10] (e.g. honeycomb panels [29]). In this section, we demonstrate how the local perturbation method can be extended to this case. We consider connected elements of periodic structure, see Fig.5. In this case, the local elastic constants $a_{i j k l}(\mathbf{y})$ are periodic with periodicity cell $Y^{-}\left(Y^{+}\right)$on the left (right) from the joint, see Fig.5. The zone $P_{0}$, where the frame penetrates the perforated medium, is the connecting unit. Evidently, we cannot specify this zone exactly (i.e. indicate the left and the rights boundaries of the zone exactly). We can only indicate how far the perturbations initiated by this zone extend. This may be done after the elasticity theory problem is solved.

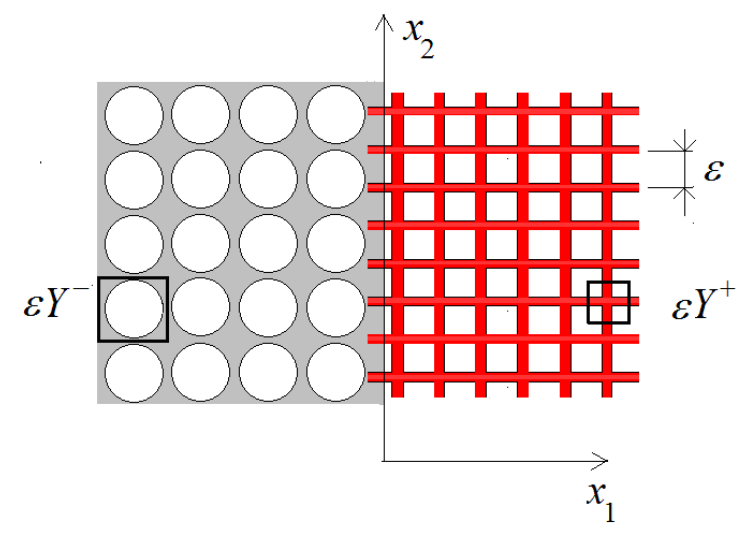

Fig.5. Connected elements of periodic structure with periodicity cell $Y^{-}\left(Y^{+}\right)$

We seek a solution to (4) in the form

$$
\mathbf{u}(\mathbf{x})=\mathbf{u}_{0}(\mathbf{x})+\varepsilon \mathbf{u}_{1}(\mathbf{x} / \varepsilon)+\varepsilon \mathbf{u}_{p}(\mathbf{x} / \varepsilon),
$$

where $\varepsilon \mathbf{u}_{1}(\mathbf{x} / \varepsilon)$ is the local perturbation corrector described by (3) and (8), and $\varepsilon \mathbf{u}_{p}(\mathbf{x} / \varepsilon)$ is the periodic corrector used in homogenization theory [27], i.e. a function with the periodicity cell $Y^{-}\left(Y^{+}\right)$on the left (right) of the joint. The periodic corrector $\varepsilon \mathbf{u}_{p}(\mathbf{x} / \varepsilon)$ is used to describe the local SSS in the main parts of joined periodic structures. The local perturbation corrector $\varepsilon \mathbf{u}_{1}(\mathbf{x} / \varepsilon)$ is used to describe both the local SSS in the connecting unit and SSS in the BLs. 
The trial functions $\mathbf{v}(\mathbf{x})$ has a similar representation to $\mathbf{u}(\mathbf{x})$ and may be expressed as

$$
\mathbf{v}(\mathbf{x})=\mathbf{v}_{0}(\mathbf{x})+\varepsilon \mathbf{v}_{1}(\mathbf{x} / \varepsilon)+\varepsilon \mathbf{v}_{p}(\mathbf{x} / \varepsilon)
$$

On inserting (24), (25) into (4), one arrives at a variational equation with respect to the function $\mathbf{u}_{1}(\mathbf{y})+\mathbf{u}_{p}(\mathbf{y})$, namely

$$
\int_{P} a_{i j k l}(\mathbf{x} / \varepsilon)\left(u_{1 k, l y}+u_{p k, l y}+u_{0 k, l x}\right)\left(v_{1 i, j y}+v_{p i, j y}\right) d \mathbf{x}=0, \text { for any } \mathbf{v}_{1} \in V_{1}, \mathbf{v}_{p} \in V_{p},
$$

where $\mathbf{v}_{p} \in V_{p}$ denotes the set of $H_{2}^{1}(P)$ functions periodic with the periodicity cell $Y^{-}\left(Y^{+}\right)$on the left (right) from the joint.

Equation (26) is equivalent to the following BVP

$$
\left\{\begin{array}{c}
\left(a_{i j k l}(\mathbf{y})\left(u_{1}+u_{p}\right)_{k, l y}+a_{i j \alpha \beta}(\mathbf{y}) u_{0 \alpha, \beta x}(\mathbf{x})\right)_{, j y}=0 \quad \text { in } P, \\
\left(a_{i j k l}(\mathbf{y})\left(u_{1}+u_{p}\right)_{k, l y}+a_{i j \alpha \beta}(\mathbf{y}) u_{0 \alpha, \beta x}(\mathbf{x})\right) n_{j}=0 \text { on } S, \\
\mathbf{u}_{1}(\mathbf{y})+\mathbf{u}_{p}(\mathbf{y}) \text { tends to function periodic with periodicity cell } Y^{-} \text {as } y_{1} \rightarrow-\infty \\
\text { and with periodicity cell } Y^{+} \text {as } y_{1} \rightarrow \infty .
\end{array}\right.
$$

The BVP (27)-(29) does not have any solution for arbitrary $u_{0 \alpha, \beta x}(\mathbf{x})$. Let us derive the solvability condition for the BVP (27)-(29). Integrating by parts equation (27), and respecting conditions (28), (29), one obtains

$$
\int_{y_{1}=-\infty} \boldsymbol{\sigma}_{n} d y_{2}+\int_{y_{1}=+\infty} \boldsymbol{\sigma}_{n} d y_{2}=0
$$

It is known that (see, e.g. [27]) $\int_{y_{1}=-\infty(+\infty)} \boldsymbol{\sigma}_{n} d y_{2}=A_{i 1 \alpha \beta}^{-(+)} e_{\alpha \beta}^{-(+)}$, where $A_{i j \alpha \beta}^{-}$and $A_{i j \alpha \beta}^{+}$are the homogenized elastic constants of the bodies placed on the left and on right of the joint. Then we obtain the solvability condition for the BVP (27)-(29)in the form

$$
A_{i 1 \alpha \beta}^{-} e_{\alpha \beta}^{-}=A_{i 1 \alpha \beta}^{+} e_{\alpha \beta}^{+} \text {. }
$$

As above, $e_{\alpha \beta}^{-}$and $e_{\alpha \beta}^{+}$denote the elastic strains corresponding to the displacements $\mathbf{u}_{0}(\mathbf{x})$ associated with the main part of the connected elements.

The compatibility condition (31) is independent from the properties of the joint. As in the case of homogeneous materials (see Sect.2), the solvability conditions (31) can be used to show that it is not possible to separate the fast and slow variables within the BVP (27)-(29). One can however express $e_{\alpha \beta}^{-}$through $e_{\alpha \beta}^{+}$(or vice versa) and formulate a problem in which the separation of fast and slow variables is possible.

For large $\left|y_{2}\right|$ the function $\mathbf{u}_{1}(\mathbf{y})$ tends to zero, hence $\mathbf{u}_{1}(\mathbf{y})+\mathbf{u}_{p}(\mathbf{y}) \approx \mathbf{u}_{p}(\mathbf{y})$ and the BVP (27)-(29) in the main parts of the joined structures takes the form 


$$
\left\{\begin{array}{l}
\left(a_{i j k l}(\mathbf{y}) u_{p k, l y}+a_{i j \alpha \beta}(\mathbf{y}) u_{0 \alpha, \beta x}(\mathbf{x})\right)_{, j y}=0 \quad \text { in } P^{-}\left(P^{+}\right), \\
\left(a_{i j k l}(\mathbf{y}) u_{p k, l y}+a_{i j \alpha \beta}(\mathbf{y}) u_{0 \alpha, \beta x}(\mathbf{x})\right) n_{j}=0 \text { on } S \\
\mathbf{u}_{p}(\mathbf{y}) \text { is periodic with periodicity cell } Y^{-}\left(Y^{+}\right) .
\end{array}\right.
$$

The BVP (32), (33) denotes, in fact, two BVPs, one for $y_{1}<0$ and another for $y_{1}>0$. Each of these problems may be regarded as a standard problem within homogenization theory and separation of slow and fast variables can be used (note, there are no solvability conditions on $u_{0 \alpha, \beta x}(\mathbf{x})$ in this case). In the procedure of separation of slow and fast variables, one considers $u_{0 \alpha, \beta x}(\mathbf{x})$ as a parameter and, taking into account the linearity of the problem, we write the following Ansatz for solution to BVP (32), (33) in $P^{-}\left(P^{+}\right)$:

$$
\mathbf{u}_{p}(\mathbf{y})=\mathbf{N}^{\alpha \beta}(\mathbf{y}) u_{0 \alpha, \beta x}(\mathbf{x}),
$$

where $N^{\alpha \beta}(\mathbf{y})$ are solutions to the periodicity cell problems

$$
\left\{\begin{array}{l}
\left(a_{i j k l}(\mathbf{y}) N_{k, l y}^{\alpha \beta}+a_{i j \alpha \beta}(\mathbf{y})\right)_{, j y}=0 \text { in } Y^{-}\left(Y^{+}\right), \\
a_{i j k l}(\mathbf{y}) N_{k, l y}^{\alpha \beta}+a_{i j \alpha \beta}(\mathbf{y}) n_{j}=0 \text { on } S, \\
\mathbf{N}^{\alpha \beta}(\mathbf{y}) \text { is periodic with periodicity cell } Y^{-}\left(Y^{+}\right) .
\end{array}\right.
$$

Note that in the representation (34), we use in $P^{-} \mathbf{u}_{0}$ computed for $x_{1}<0$ and $\mathbf{N}^{\alpha \beta}$ computed for $Y^{-}$; in $P^{+}$, we use $\mathbf{u}_{0}$ computed for $x_{1}>0$ and $\mathbf{N}^{\alpha \beta}$ computed for $Y^{+}$.

We also note the phenomenon of "erasing" of the individual properties of the joint. The potential energy of the joined structures

$$
E=\frac{1}{2} \int_{P} a_{i j k l}(\mathbf{x} / \varepsilon) u_{k, l y}^{2} d \mathbf{x}+\int_{P} \mathbf{q}(\mathbf{x}) \mathbf{u} d \mathbf{x}
$$

can be written, by taking account of (34), as follows

$$
\begin{gathered}
E=\frac{1}{2} \int_{P}\left[a_{i j k l}(\mathbf{x} / \varepsilon)\left(u_{1 k, l x}(\mathbf{x} / \varepsilon)+N_{k, l x}^{\alpha \beta}(\mathbf{x} / \varepsilon) u_{0 \alpha, \beta x}(\mathbf{x})+u_{0 k, l x}(\mathbf{x})\right)^{2}\right] d \mathbf{x}+ \\
\int_{P}\left[\varepsilon \mathbf{q}(\mathbf{x}) \mathbf{u}_{1}(\mathbf{x} / \varepsilon)+\mathbf{q}(\mathbf{x})\left(\varepsilon \mathbf{N}_{k, l x}^{\alpha \beta}(\mathbf{x} / \varepsilon) u_{0 \alpha, \beta x}(\mathbf{x})+\mathbf{u}_{0}(\mathbf{x})\right)\right] d \mathbf{x} .
\end{gathered}
$$

The local perturbation $\mathbf{u}_{1}(x / \varepsilon)$ takes values sufficiently different from zero only in the $\varepsilon$ vicinity of the joint. It means that for small $\varepsilon$, the integrals in equation (36), containing the local perturbation $\mathbf{u}_{1}(\mathbf{x} / \varepsilon)$, may be neglected. The term $\mathbf{q}(\mathbf{x}) \varepsilon \mathbf{N}_{k, l x}^{\alpha \beta}(\mathbf{x} / \varepsilon) u_{0 \alpha, \beta x}(\mathbf{x})$ in (36) may also be neglected. Hence, the functional $E$ tends to 


$$
E_{0}=\frac{1}{2} \int_{P} A_{i j k l}(\mathbf{x}) u_{0 k, l x}^{2} d \mathbf{x}+\int_{P} \mathbf{q}(\mathbf{x}) \mathbf{u}_{0} d \mathbf{x}
$$

where

$$
A_{i j k l}(\mathbf{x})= \begin{cases}A_{i j k l}^{-} & \text {for } x_{1}<0, \\ A_{i j k l}^{+} & \text {for } x_{1}>0\end{cases}
$$

are the homogenized elastic coefficients, defined as

$$
\begin{gathered}
A_{i j k l}^{-}=\frac{1}{2} \int_{Y^{-}} a_{m n p q}(\mathbf{y})\left(N_{m, n y}^{i j}(\mathbf{y})+\delta_{i m} \delta_{j n}\right)\left(N_{p, q y}^{k l}(\mathbf{y})+\delta_{k p} \delta_{l q}\right) d \mathbf{y} \\
A_{i j k l}^{+}=\frac{1}{2} \int_{Y^{+}} a_{m n p q}(\mathbf{y})\left(N_{m, n y}^{i j}(\mathbf{y})+\delta_{i m} \delta_{j n}\right)\left(N_{p, q y}^{k l}(\mathbf{y})+\delta_{k p} \delta_{l q}\right) d \mathbf{y} .
\end{gathered}
$$

In (38), we use proper function $\mathbf{N}^{\alpha \beta}$ in $Y^{-}$and $Y^{+}$.

Formula (37) defines the potential energy of the joined homogenized structures. The principle of minimal potential energy for these structures leads to the equation

$$
\delta E_{0}=0
$$

where $\delta E_{0}$ denotes an infinitesimal variation of the potential energy. The variational equation (39) leads to the homogenized equations of equilibrium of the homogenized structures and the macroscopic conditions on the joint. Let us formulate conditions on the joint. As the displacements are continuous, then

$$
\left[\mathbf{u}_{0}(\mathbf{x})\right]=0,
$$

where $[f(\mathbf{x})]=f^{+}(\mathbf{x})-f^{-}(\mathbf{x})$ denotes the jump at the joint $S$ of connected structures. From (39) and (37), (38), (40), we obtain the following condition

$$
\left[A_{i j k l}(\mathbf{x}) u_{0 k, l x}\right] n_{j}=0 .
$$

The macroscopic condition (41) on the joint involves the homogenized elastic constants $A_{i j k l}(\mathbf{x})$ of both the left and the right bodies. These constants depend only on the microstructure of the main parts of the connected bodies, see (35) and (38). This indicates that the macroscopic conditions on the joint are completely independent of the properties of the joint.

It is also remarked that although the contribution of the local perturbation $\varepsilon \mathbf{u}_{1}(x / \varepsilon)$ to the potential energy is small, the derivatives of $\varepsilon \mathbf{u}_{1}(x / \varepsilon)$ play a significant role within the local SSS in the joint zone. In the vicinity of joint, according to (24), we obtain

$$
\sigma_{i j}=a_{i j k l}(\mathbf{x} / \varepsilon)\left[u_{0 k, l x}(\mathbf{x})+u_{1 k, l y}(\mathbf{x} / \varepsilon)+u_{p k, l y}(\mathbf{x} / \varepsilon)\right]
$$


In the main parts of the connected bodies $\mathbf{u}_{1}(\mathbf{y}) \approx 0$ and, in consequence, (34) therefore takes the form

$$
\sigma_{i j}=a_{i j k l}(\mathbf{x} / \varepsilon)\left[\delta_{k \alpha} \delta_{l \beta}+N_{k, l y}^{\alpha \beta}(\mathbf{x} / \varepsilon)\right] u_{0 \alpha, \beta x}(\mathbf{x}),
$$

coinciding with predictions of standard homogenization theory [3, 5, 27].

\section{Example 2. Stress concentration in the seam formed by explosion welding of a homogeneous material and a layered composite}

In this section, we present an application of our method to calculate the local SSS in a seam formed by explosion welding of a homogeneous material and a layered composite. We consider a homogeneous material copper (on the left); which is connected by explosion welding to a layered aluminum composite, reinforced with aluminum oxide (right), as displayed in Fig.1. The elastic characteristics of the aluminum oxide are $E=85 \mathrm{GPa}, v=0.3$.

We assume that the composite in its "main part" has a periodic structure in the direction of the $O x_{1}$-axis, formed by repetition of layers of aluminum and aluminum oxide. The period of the composite has the form "layer of aluminum - a layer of aluminum oxide", with length $5 \mathrm{~mm}$. The volume fractions of the aluminum and aluminum oxide are 0.9 and 0.1 , respectively. We assume that near the welding seam the layers are bent in such way that the volume fractions of aluminum and aluminum oxide is saved as indicated above.

As in the previous example, it is assumed that the material interface has the sinusoidal shape (22) with parameters (23). The results of numerical computations are presented in Figs 6-8.

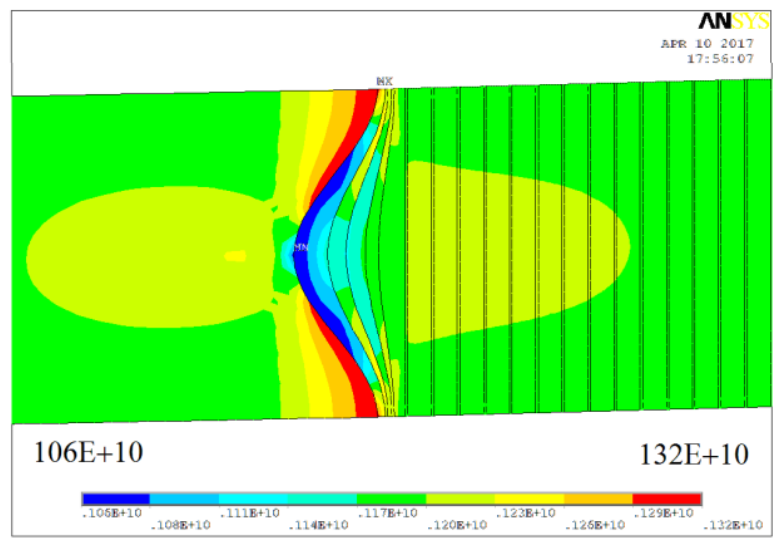

Fig.6. Shear-type deformation (deformed periodicity cell and local von Mises stress) (values on the scale vary from $0.106 E+10$ on lhs to $0.132+10$ on rhs with step $0.03 E+10$, the first step is

$$
0.002 E+10)
$$




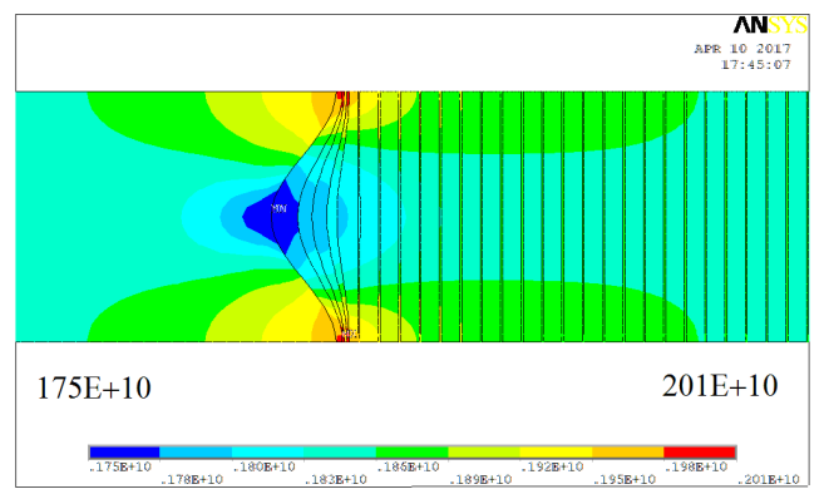

Fig.7. Extension in direction Ox-axis (deformed periodicity cell and local von Mises stress) (values on the scale vary from $0.175 \mathrm{E}+10$ on lhs to $201+10$ on rhs with step $0.03 \mathrm{E}+10$, the second step is $0.002 \mathrm{E}+10$ )

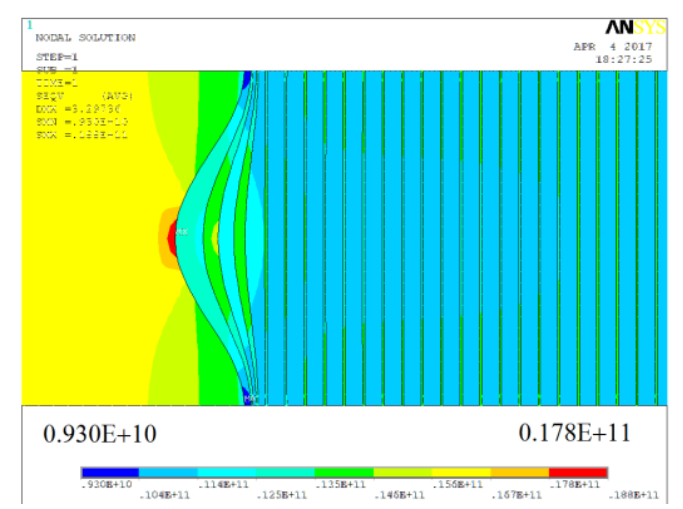

Fig.8. Extension in direction Oy-axis (deformed periodicity cell and local von Mises stress) (values on the scale vary from $0.93 E+10$ on lhs to $0.178+11$ on rhs with step 0.010-0.011E+11)

We define the von Mises stress concentration as $k=\sigma(\mathbf{x}) / \sigma_{\infty}$, where $\sigma(\mathbf{x})$ is the von Mises stress at the point $\mathbf{x}$ and $\sigma_{\infty}$ is von Mises stress "at infinity" (i.e. in the main parts of the structure). It is seen from the Figs. 6-8 that in both the weld seam and BLs, the von Mises stress concentration does not exceed $10 \%$. The implication is that explosion welding yields a seam that does not create significant stress concentrators. The bearing capacity of the structure is not therefore reduced significantly in comparison to the bearing capacities of the materials to be welded. In Table 1 the component of the structure in which the maximum of $k$ occurs is indicated.

Table 1. Maximum of von Mises stress concentration $k=\sigma_{\max } / \sigma_{\infty}$.

\begin{tabular}{|l|c|l|}
\hline Macro deformation type & $k$ & component \\
\hline Extension in the direction $O x_{1}$ & 1.08 & aluminum oxide \\
\hline
\end{tabular}




\begin{tabular}{|l|c|l|}
\hline Extension in the direction $\mathrm{Ox}_{2}$ & 1.09 & copper \\
\hline Shear & 1.08 & copper \\
\hline
\end{tabular}

\section{Isolation of a local perturbation - the problem of representative element for joint}

For a model based on the concept of a connecting unit, or transition zone, it is impossible to clearly separate the joint from the joined elements. On the other hand, the corresponding SSSs are the "local objects" and have a characteristic size that is supported by our numerical calculations. So we can determine the minimal fragment of the connected elements adjacent to the connecting unit so that the solution in the selected zone approximates the solution of the original problem with sufficient accuracy. We call such an object the "representative element for joint". The selection of the representative element for joint allows one to efficiently use numerical approaches.

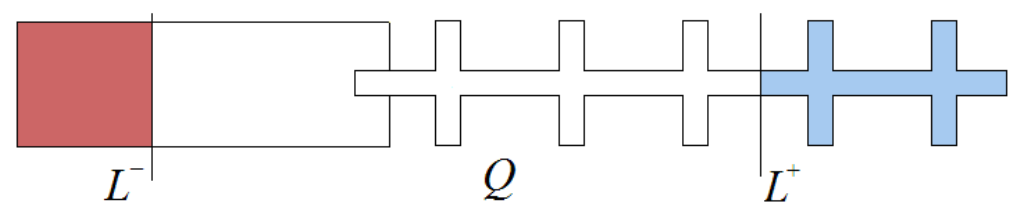

Fig.9. Finite fragment of structure containing the joint

Consider a domain $Q$ containing the joint unit $P_{0}$ inside. Take $Q$ in such way that both the left $L^{-}$and right $L^{+}$boundaries are "far enough" from the connecting unit. We may therefore assume that $\mathbf{u}_{1}(\mathbf{y}) \approx 0$ outside $Q$ and, in particular, on the lines $L^{-}$and $L^{+}$. The meaning of the perhaps rather vague term "far enough" will be discussed and clarified below.

Let us describe using concept of "representative element for joint" for numerical solution. Let us introduce the function $\mathbf{Z}(\mathbf{y})=\mathbf{u}_{p}(\mathbf{y})+y_{\alpha} \mathbf{e}_{\beta} u_{0 \alpha, \beta x}(\mathbf{x})$. Using (34), one obtains

$$
\mathbf{Z}(\mathbf{y})=\mathbf{N}^{\alpha \beta}(\mathbf{y}) u_{0 \alpha, \beta x}(\mathbf{x})+y_{\alpha} \mathbf{e}_{\beta} u_{0 \alpha, \beta x}(\mathbf{x})=\left(\mathbf{N}^{\alpha \beta}(\mathbf{y})+y_{\alpha} \mathbf{e}_{\beta}\right) u_{0 \alpha, \beta x}(\mathbf{x}) .
$$

We recall that both that $\mathbf{u}_{0}(\mathbf{x})$, the solution to the homogenized problem, and $\mathbf{N}^{\alpha \beta}(\mathbf{y})$, the solution to the cell problem, are known functions. We now wish to introduce $\mathbf{P}(\mathbf{y})=\mathbf{u}_{1}+\mathbf{u}_{p}+y_{\alpha} \mathbf{e}_{\beta} u_{0 \alpha, \beta x}(\mathbf{x})$. On the lines $L^{-}$and $L^{+}, \mathbf{u}_{1}+\mathbf{u}_{p}=\mathbf{u}_{p}$, and so $\mathbf{P}(\mathbf{y})=\mathbf{Z}(\mathbf{y})$. Replacing the conditions at infinity (29) with the conditions on the boundaries $L^{-}$and $L^{+}$, we obtain the following BVP for $\mathbf{P}(\mathbf{y})$ 


$$
\left\{\begin{array}{l}
\left(a_{i j k l}(\mathbf{y}) P_{k, l y}\right)_{, j y}=0 \quad \text { in } Q \\
a_{i j k l}(\mathbf{y}) P_{k, l y} n_{j}=0 \text { on } S, \\
\mathbf{P}(\mathbf{y})-y_{\alpha} \mathbf{e}_{\beta} u_{0 \alpha, \beta x}(\mathbf{x}) \text { is periodic in } y_{2} \text { with period } T, \\
\mathbf{P}(\mathbf{y})=\left(\mathbf{N}^{\alpha \beta}(\mathbf{y})+y_{\alpha} \mathbf{e}_{\beta}\right) u_{0 \alpha, \beta x}(\mathbf{x}) \text { on } L^{-} \\
\mathbf{P}(\mathbf{y})=\left(\mathbf{N}^{\alpha \beta}(\mathbf{y})+y_{\alpha} \mathbf{e}_{\beta}\right) u_{0 \alpha, \beta x}(\mathbf{x}) \text { on } L^{+}
\end{array}\right.
$$

The solution of the BVP (45), (46) defines the local SSS in both the joint unit $P_{0}$ and in the adjacent fragments of the structural elements. Before progressing, we will discuss the previously used term "far enough distance". In order to provide a quantitative estimate, it is necessary to estimate the length of BLs decay zone. We define the length of the BL decay zone by the condition of 10-times decreasing in intensity. Taking into account exponential decay within the BLs, other definitions of the length of BL decay zone (say e-times or 100-times decreasing) lead to similar values. Theoretically, exponential estimations of the length of BL decay zones have previously been obtained, e.g., in [23]. However, estimates from [23] are not informative for practice and it is natural to use numerical experiments to estimate the BL decay zone.

Analysis of numerical solutions show that if the mechanical properties of the materials of the connected bodies and joint (ratios of identical mechanical properties) do not exceed 10, the length of BLs decay zone (i.e., the "far enough distance") can be estimated as 3-5 the "thickness" of the periodicity cell (note that similar estimates have previously reported for thermal problems in [25]). The calculations in the examples presented in Secs 3 and 5 were carried out by solving the BVP (45), (46) with the "far enough distance" equal 5 maximal size of the periodicity cell.

For connected structures with high contrast components, the above-mentioned estimate does not work. As an example we cite high contrast layered composites with periodically alternating soft and hard layers, for which the shear-lag model predicts the characteristic length $L_{c}$ of the BLs is given by

$$
L_{c} \approx K h \sqrt{E_{2} / E_{1}}
$$

see [21], where $K$ is a numerical coefficient $(K=10 \ldots 20), h$ is the thickness of the soft layer, $E_{1}, E_{2}$ are the rigidities of the soft and the hard layer. The above formula has been recommended for use if $E_{2} / E_{1} \geq 100$ [15]. We also note that estimation (47) was supported by experimental data [11].

\section{Example 3. Lattice-to-solid connection}


In Examples 1 and 2, it is straight forward to find the solutions $\mathbf{N}^{\alpha \beta}(\mathbf{y})$ of the cell problems (34), (35) in the main part of the connected materials. For homogeneous materials, they are zero, for layered materials they depends only on the variable $y_{1}$. In this section we present the results of numerical calculations for the lattice-to-solid system (Fig. 9.10), when $\mathbf{N}^{\alpha \beta}$ (y) are essentially two-dimensional. The solid material is aluminum $(E=70 G P a, v=0.34)$, the lattice material is steel ( $E=300 G P a, v=0.28$ ) and there is an ideal contact between the materials, see Fig. 10 .

Figs 10-12 present the von Mises stress for basic types of plane strain $e_{\alpha \beta}=\delta_{\alpha \beta}, \alpha, \beta=1,2$ extension in direction $O x$ - and $O y$-axis and shear.

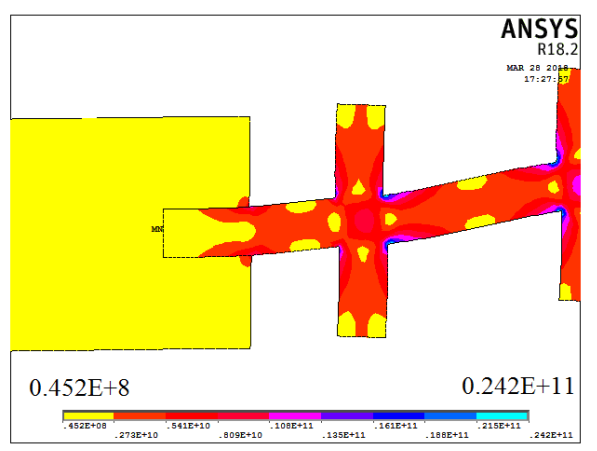

Fig.10. Shear-type deformation (deformed periodicity cell and local von Mises stress) (values on the scale vary from $0.452 E+8$ on lhs to $0.242+11$ on rhs with step $0.03 E+11$ )

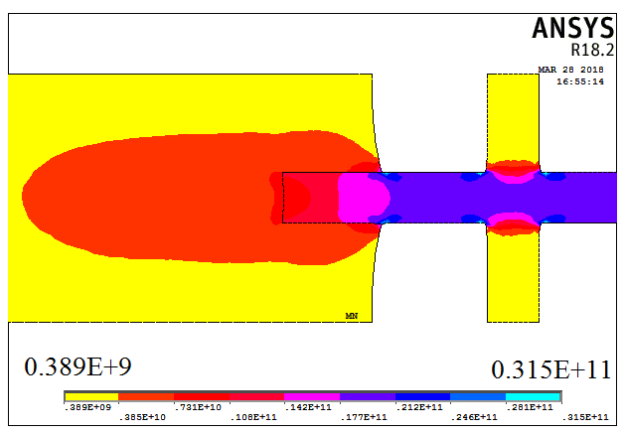

Fig.11. Extension in direction Ox -axis (deformed periodicity cell and local von Mises stress) (values on the scale vary from $0.389 E+9$ on lhs to $0.315+11$ on rhs with step $0.03 E+11$ )

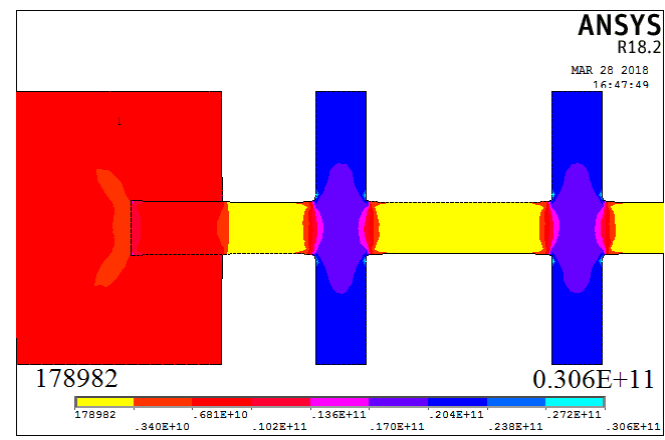


Fig.12. Extension in direction Oy-axis (deformed periodicity cell and local von Mises stress)

(values on the scale vary from 178982 on lhs to $0.306+11$ on rhs with step $0.034 E+11$ )

The results presented in this Section, leads to solution to the following closely connected problems.

The problem of insulation of specific connecting unit: Which joint in a structure may be considered with good accuracy without accounting for other joints? In accordance with our analysis, the distance from the joint to the other joints must not be less than 10 characteristic sizes of the connecting unit. If the distances between the joints are less, the joints must be aggregated in an integrated joint and considered as one joint. For the system of insulated connecting units, representation (3) is changed to the following:

$$
\mathbf{u}^{0}(\mathbf{x})+\sum_{i=1}^{n} \varepsilon \mathbf{v}_{i}\left(\left(\mathbf{x}-\mathbf{x}_{i}\right) / \varepsilon\right)
$$

where $\varepsilon \mathbf{v}_{i}\left(\left(\mathbf{x}-\mathbf{x}_{i}\right) / \varepsilon\right)$ denotes the local perturbation corresponding to the connecting unit placed at $\mathbf{x}=\mathbf{x}_{i}$. The functions $\mathbf{v}_{i}(\mathbf{y})$ may be computed independently one from other and depend on $\operatorname{grad} \mathbf{u}^{0}\left(\mathbf{x}_{i}\right)$ as parameter.

The influence of the connecting units on the global stiffness: How joints influence the overall stiffness of the system "in whole". The effect of "erasing" of non-degenerated joints means that joints do not influence the global stiffness as $\varepsilon \rightarrow 0$. The condition $\varepsilon \rightarrow 0$ is a mathematical idealization. In reality, $\varepsilon$ is small, and the corrector (19) has the order $\varepsilon^{-n+1}(2 H+D)$.

The problem of equivalent (representative) fragment of structure, containing insulated connecting units: In this case the joint can not be considered in isolation. On the other hand, from the computational point of view, it is difficult to consider the whole structure. What is the size of the fragment of the structure that provides sufficient accuracy of calculation? In accordance with our analysis, this fragment must have length not less than 10 characteristic sizes of the connecting unit.

\section{Concluding remarks}

Fundamental problems concerning the analysis of connected bodies by systems of connecting units have been considered. Under the assumption that the connecting units have a relatively small size compared with the objects being connected, a two-scale asymptotic method was applied to the problem. The method of local perturbations was employed, which allowed us to 
analyse the classical rapidly decaying BLs, together with the local SSS in the connecting unit, in a uniform way.

It was found that the problem of connection allows an asymptotic decomposition. At a macroscopic level, an interface problem, with appropriate interface conditions, was seen to arise. The interface conditions are completely independent of the properties of the joint. The properties of the joint manifest themselves within the next step of the asymptotic decomposition. This step leads to a problem relating to a connecting unit subjected to a uniform field. The connecting unit accounts for all individual properties of the joint, with the "uniform field" determined from the solution to the macroscopic problem. The local SSS in the vicinity of the joint consists of the SSS in the connecting unit, together with the rapidly decaying BLs in the connected elements. The local SSS in the connecting unit may only be determined by the specific structure of this unit; it cannot be described within the frameworks of the BLs approach.

Motivated by our asymptotic analysis, a numerical method for simultaneously calculating the SSS in the connected structures and the connecting unit has been developed. An illustrative examples, computation of the SSS in the vicinity of an explosion welding seem and lattice-tosolid connection, were been presented. For numerical calculations, it is convenient to use a certain bounded domain, rather than satisfying the decay conditions at infinity. We determine the minimal fragments of the connected elements adjacent to the connecting unit so that the solution in the selected zone approximates the solution of the original problem with sufficient accuracy. The length of the fragments of the connected elements must be not less than the length of the BLs decay. For "non-degenerated" joints, the length of BLs decay zone is estimated as 35 the "thickness" of the periodicity cell associated with the connecting unit.

On the basis of the theoretical analysis, we propose a numerical procedure that can be carried out using standard FEM codes. An algorithm assumes two-step computations. First, the interface problem is solved. After which, local problems for connecting units, subjected to the uniform stress/strain field determined during the first step, are solved for the typical connecting unit(s). Within this work we presented examples of the calculation of SSS in the vicinity of a seam arising during explosion welding of homogeneous and composite materials. However, the developed approach may be applied more generally to elucidate connection phenomena of both homogeneous and heterogeneous (e.g., composite) bodies.

\section{References}

[1] G. Allaire, M. Amar, Boundary layer tails in periodic homogenization, ESAIM Control Optim. Calc. Var. 4 (1999) 209-243. 
[2] B.D. Annin, Ye.B. Karpov, Elements of Mechanics of Composites (Novosibirsk State University Publ., Novosibirsk, 2016) (in Russian).

[3] N.S. Bakhvalov, G.P. Panasenko, Averaging Processes in Periodic Media. Mathematical Problems in Mechanics of Composite Materials (Kluwer, Dordrecht, 1989).

[4] S.M. Bauer, S.B. Filippov, A.L. Smirnov, P.E. Tovstik, R. Vaillancourt Asymptotic Methods in Mechanics of Solids (Birkhäuser, Basel, 2015).

[5] A. Bensoussan, J.-L. Lions, G. Papanicolau, Asymptotic Analysis for Periodic Structures (North-Holland, Amsterdam, 1978).

[6] A. Bensoussan, J.-L. Lions, G. Papanicolau, Boundary layers and homogenization of transport processes. Publ. Res. Inst. Math. Sci. Kyoto Univ. 15 (4) (1979) 53-157.

[7] V.L. Berdichevsky, Variational Principles of Continuum Mechanics: I. Fundamentals (Springer, Berlin, 2010)

[8] J. H. Bickford, S. Nassar (Eds), Handbook of Bolts and Bolted Joints (Marcel Dekker, NY, 1998).

[9] G.P. Cherepanov, L.S. Rybakov, Design of riveted panels. Soviet App. Mech., 13(10) (1977) 745-748.

[10] Composite Materials and Joining Technologies for Composites, Vol. 7: Proc. Annual Conf. Exp. Appl. Mech., E. Patterson, D. Backman, G. Cloud (Eds) (Springer, NY, 2012).

[11] A.G. Demeshkin, A.G. Kolpakov, Experimental verification of the shear model for the calculation of structures from a unidirectional composite, Dynamics of Continuum. Novosibirsk, M.A. Lavrent'ev Institute of Hydrodynamics of Siberian Branch of the USSR Academy of Sc. No. 55, (1982) 128-132 (in Russian).

[12] H. Dumontet, Study of a boundary layer problem in elastic composite materials, ESAIM Math. Model. Numer. Anal. 20 (1986) 265-286.

[13] G. Duvaut, Analyse Fonctionnelle et mécanique des milieux continus. Application à l'étude des Matériaux composites élastiques à structure périodique-homogénéisation. In: W.T. Koiter (Ed.) Theoretical and Applied Mechanics (North-Holland, Amsterdam, 1976) $110-132$.

[14] Joining Technologies for Composites and Dissimilar Materials, Proc. Annual Conf. Exp. Appl. Mech. 10, G.L. Cloud, E. Patterson, D. Backman (Eds) (Springer, NY, 2016).

[15] A.L. Kalamkarov, A.G. Kolpakov, Analysis, Design and Optimization of Composite Structures (Wiley, Chichester, New York, 1997).

[16] A.G. Kolpakov, I.V. Andrianov, Asymptotic decomposition in the problem of joined elastic beams, ZAMM 94 (26) (2014) 818-836. 
[17] A.G. Kolpakov, A. Gaudiello, Influence of non degenerated joint on the global and local behavior of joined rods, Int. J. Engng Sci. 49 (9) (2011) 295-309.

[18] V.A. Kozlov, V.G. Maz'ya, A.B. Movchan, Asymptotic Analysis of Fields in MultiStructures (Oxford University Press, Oxford, 1999).

[19] G. Lin, X.G. Meng, A. Cornec, K.H. Schwalbe, The effect of strength mismatch on mechanical performance of weld joints, Int. J. Fracture 96 (4) (1999) 37-54.

[20] A.B. Movchan, Multi-structures: asymptotic analysis and singular perturbation problems, European J. Mech. A/Solids 25 (2006) 677-694.

[21] M. Neuss-Radu, A result on the decay of the boundary layers in the homogenization theory, Asymp. Anal. 23 (2000) 313-328.

[22] O.A. Oleynik, A.S. Shamaev, G.A.Yosifian, Mathematical Problems in Elasticity and Homogenization (North-Holland, Amsterdam, 1992).

[23] G.P. Panasenko, Higher order asymptotics of solutions of problems on the contact of periodic structures, Mathematics of the USSR-Sbornik 38(10) (1981) 465-494.

[24] G.P. Panasenko, Multi-Scale Modeling for Structures and Composites (Springer, Berlin, 2005).

[25] W. Pietraszkiewicz, V. Konopicska, Junctions in shell structures: A review, Thin-Walled Structures 95 (2015) 310-334.

[26] E.C. Rodabaugh, T.J. Atterbury, Stresses in tapered transition joints in pipelines and pressure vessels. J. Eng. Ind. 84(9) (1962), 321-328.

[27] E. Sanchez-Palencia, Non-Homogeneous Media and Vibration Theory (Springer, Berlin, New York, 1980).

[28] E. Sanchez-Palencia, Boundary layers and edge effects in composites. In: Homogenization Techniques for Composite Materials. Sanchez-Palencia, E., Zaoui, A. (Eds.) (Springer, Berlin, New York, 1987) 122-193.

[29] A.I. Yendogur, M.V.Vainberg, K.M. Ierusalimskyi, Honeycomb sandwiches. Choice of parameters and designing (Moscow, Mashinostriyenie, 1986) (in Russian).

[30] K. Washizu, Variational Methods in Elasticity and Plasticity. 3rd Ed. (Pergamon Press, Oxford, 1982).

[31] V.V. Zhikov, Homogenization of elasticity problems on singular structures, Izvestia Math. 66 (5) (2002) 299-365.

[32] V.V. Zhikov, S.E. Pastukhova, Homogenization for elasticity problems on periodic networks of critical thickness, Sbornik Math. 194 (8) (2003) 697-732. 